\title{
Efeito do Tempo Pós-Queimada Sobre Comunidades de Tephritidae (Diptera) em Áreas de Cerrado na Chapada dos Guimarães - MT
}

\author{
Kellen Fávero ${ }^{\circledR}$, Leandra Bordignon ${ }^{2}$, Kleber Vecchi Junior ${ }^{2}$ \& Soraia Diniz² \\ 1. Universidade Federal da Grande Dourados, e-mail: kellenfavero@yahoo.com.br (Autor para correspondência ${ }^{\bowtie}$ ). 2. Universidade Federal do Mato \\ Grosso, e-mail: 1b_bio@hotmail.com, klebermano@hotmail.com, saf@cpd.ufmt.br.
}

\section{EntomoBrasilis 3 (2): 29-33 (2010)}

\begin{abstract}
Resumo. Este trabalho teve como objetivos: (i) analisar a riqueza, a abundância e a ocorrência de espécies de insetos endófagos de capítulos de Asteraceae da Chapada dos Guimarães-MT; (ii) avaliar o efeito do tempo decorrido pós-queimada sobre riqueza, abundância e distribuição de espécies de Tephritidae endófagos. Foram amostradas 13 áreas de cerrado nas quais foi traçado um transecto de 250m x 2m sobre uma isoclina topográfica. Todos os capítulos, de cada indivíduo florido, foram coletados e mantidos em potes telados para criação. Um total de 341 indivíduos pertencentes a sete espécies emergiram dos capítulos. Cecidochares e Xanthaciura foram os gêneros mais bem representados em relação ao número de espécies. A maioria dos insetos emergiu de capítulos coletados em apenas uma localidade. Somente Xanthaciura chrysura (Thomson) ocorreu em todas as localidades amostradas em que emergiram insetos. O tempo decorrido após a última queimada não teve efeito significativo sobre a riqueza, abundância e distribuição das espécies de Tephritidae endófagos.
\end{abstract}

Palavras-chave: Asteraceae; Interação inseto-planta; Fogo; Planta hospedeira; Riqueza

\section{Effect of Post-Fire Time on Tephritidae (Diptera) Communities in Cerrado Areas at Chapada dos Guimarães - MT}

Abstract. The objectives of this study were to: (i) analyze the richness, abundance, and occurrence of endophagous insects in capitula of Asteraceae at Chapada dos Guimarães; (ii) evaluate the effect of post-fire time on the richness, abundance, and distribution of endophagous Tephritidae species. Thirteen cerrado areas were sampled, onto which a $250 \mathrm{~m} \times 2 \mathrm{~m}$ transect was established over a topographic isocline. All capitula from individual plants were collected and maintained in screened rearing pots. Three hundred and forty-one individuals belonging to seven species emerged from the flowerheads. Cecidochares and Xanthaciura were the best-represented genera in relation to the number of species. Most insects emerged from capitula collected at a single locality. Only Xanthaciura chrysura (Thomson) occurred in all of the sampled localities at which emerged insects were obtained. The time elapsed after the last fire did not have a significant effect on the richness, abundance, and distribution of endophagous Tephritidae species.

Keywords: Asteracea; Fire; Host plant; Insect-plant interaction; Richness

A associação de plantas e insetos herbívoros é uma das interações ecológicas mais importantes na natureza (PRADO \& LEWINSOHN 2000), pois artrópodes herbívoros mantém muitos e importantes processos dos ecossistemas terrestres (DiDHAM et al. 1996), tornando-os chave para se compreender a estrutura de biodiversidade global (LEWINSOHN \& RosLim 2008).

O sistema Asteraceae-insetos associados está bem representado nos cerrados brasileiros (AlmeIDA et al. 2006; Diniz 2003; Kubota et al. 2007, Prado et al. 2002; 2004). Nestes ambientes, ocorre alta diversidade, tanto de insetos endófagos como de plantas hospedeiras que concentram alimento e abrigo para esses insetos (Lewinsohn 1991).

Tephritidae é uma das famílias com grande riqueza e abundância de espécies entre os que se alimentam de capítulos em regiões temperadas (FoOTE et al. 1993) e no Brasil (PRADO et al. 2002). O que se sabe sobre Tephritidae Neotropical é ainda muito incompleto e está mais desenvolvido para grupos de relevância econômica. Há pouca informação sobre a biologia de muitas espécies e ainda há muitas questões a serem resolvidas em relação à taxonomia deste grupo (Foote 1980; Foote et al. 1993; Prado et al. 2002).

Como todos os tipos de perturbação, o fogo tem efeitos positivos em alguns organismos (Evans 1984; ANDERSON et al.
1989; PANZER 2002) e efeitos negativos sobre os outros (Bock \& Bock 1991; SwENGEL 2001). Portanto, ainda há controvérsia sobre as respostas das populações de invertebrados (SwEngel 2001; FREDERICKSEN \& FREDERICKSEN 2002).

Em ecossistemas de cerrados, o fogo é considerado a forma de perturbação mais freqüente e antiga. No entanto, seus efeitos em longo prazo sobre dinâmica das populações e estrutura das comunidades são desconhecidos (KLINK et al. 2002). Tendo conhecimento da abundância de adaptações ao fogo presente na flora do cerrado, parece razoável supor essa similar adaptação da fauna de insetos associados (DesouzA et al. 2003). O levantamento da distribuição de espécies em áreas com históricos conhecidos de queimada representa uma maneira de se avaliar efeitos do fogo na dinâmica populacional (WHELAN 1995) e suas conseqüências sobre a comunidade.

O presente estudo teve como objetivos: (i) analisar a riqueza, a abundância e a ocorrência de espécies de insetos endófagos de capítulos de Asteraceae da Chapada dos GuimarãesMT; (ii) avaliar o efeito do tempo decorrido pós-queimada sobre riqueza, abundância e distribuição de espécies de Tephritidae endófagos. A nossa hipótese é que após a queimada, a riqueza e a abundância de espécies tendem a aumentar. Isto ocorre porque Asteraceae é uma família de plantas invasoras, sendo assim, uma perturbação como o fogo vai favorecer o estabelecimento destas 
espécies. Mas, a partir de um determinado tempo o número de espécies de Asteraceae tende a se estabilizar e em longo prazo diminuir e as espécies de Tephritidae acompanhariam essa mudança.

\section{MATERIAIS E MÉTODOS}

Sistema de Estudo. Como sistema de estudo, as Asteraceae e seus insetos associados oferece uma série de vantagens (LEWINSOHN et al. 1997). Esta família compreende cerca de 1535 gêneros e aproximadamente 23.000 espécies, representando cerca de 10\% da flora mundial (BREMER 1994), com grande importância no estrato herbáceo e arbustivo de cerrados (BATALHA \& Mantovani 2001). Várias espécies são invasoras extremamente agressivas, causando prejuízo considerável a culturas e pastagens (LeWINsohn et al. 1997).

Existem vantagens de centrar estudos em insetos endófagos como: a endofagia assegura de que estes insetos estejam associados com a planta (LEWINSOHN et al. 1997); mais informações ecológicas sobre estudos de espécies, porque depois que os endófagos são amostrados, a ligação trófica com a planta hospedeira é firmada, fornecendo-nos informações sobre a estrutura da teia alimentar (Almeida et al. 2006).

Área de Estudo. As áreas de estudo localizam-se na Área de Proteção Ambiental (APA) e Parque Nacional da Chapada dos Guimarães - MT (PNCG) nos limites dos municípios de Cuiabá e Chapada dos Guimarães - MT. O tipo climático da região é caracterizado por apresentar um inverno seco, que vai de maio a outubro, e verão chuvoso, que vai de novembro a abril. A precipitação média anual varia de 1800 a $2000 \mathrm{~mm}$ (IBAMA 2007). As áreas de estudo foram selecionadas com auxílio de técnicos da Secretaria Estadual do Meio Ambiente-SEMA e Instituto Brasileiro do Meio Ambiente e dos Recursos NaturaisIBAMA, levando-se em conta o histórico de fogo de cada uma.

Amostragem no campo. Foram realizadas amostragens em áreas de Cerrado com diferentes históricos de fogo (tempo decorrido após a última queimada). Estes corresponderam a: um ano (áreas i; j; k; l); dois anos (áreas b; c); três anos (área g) quatro anos (área f; m); cinco anos (áreas a; d; h) e 15 anos (área e), destas, quatro áreas localizam-se dentro da APA e nove dentro do Parque Nacional (Tabela 1).

Um transecto de $250 \mathrm{~m} \times 2 \mathrm{~m}$ foi marcado dentro de cada uma das áreas selecionadas, seguindo uma isoclina topográfica (Magnusson et al. 2005; Costa et al. 2005). Para tal, foram utilizadas duas estacas graduadas onde uma mangueira transparente de $14 \mathrm{~m}$ teve suas extremidades fixadas. O nível foi marcado a cada 10m. As coordenadas da origem de cada transecto foram marcadas com o auxílio do GPS.

Esta metodologia está de acordo com o método de RAPELD (modificado do método de Gentry) de MAGNusson et al. (2005). Este método maximiza a probabilidade de amostrar adequadamente as comunidades biológicas. Além disso, minimiza a variação nos fatores abióticos que afetam estas comunidades já que diminui a variação interna de topografia e solo em cada parcela e permite o uso destas variáveis como preditoras das distribuições das espécies (MAGNusson et al. 2005).

Ao lado esquerdo da isoclina foi demarcado um metro, como trilha de caminhada aos pesquisadores. Ao lado direito da trilha numa faixa de dois metros de largura e $250 m$ de comprimento, foram coletados todos os capítulos de cada planta em floração e colocados em sacos plásticos para posterior criação de endófagos em laboratório.

Para cada morfotipo vegetal foram coletados exemplares, fora do transecto, como exsicatas para identificação. Estas foram devidamente prensadas e mantidas em uma estufa a $40^{\circ} \mathrm{C}$ por três dias. Em seguida foram identificadas de acordo com a literatura especializada e com o auxílio de técnicos de botânica da Universidade Federal de Mato Grosso, e conferidas pelo especialista Roberto Lourenço Esteves (UFRJ). O material está depositado no Herbário da Universidade Federal do Mato Grosso - UFMT e no Herbário do Museu Nacional da Universidade Federal do Rio de Janeiro - UFRJ.

As coletas foram realizadas entre abril e maio de 2005, pico de floração de grande parte das espécies de Asteraceae do cerrado, principalmente da tribo Eupathorieae (Almeida et al. 2005).

Criação dos insetos. Os capítulos coletados de cada planta individual foram mantidos em um recipiente de plástico com tampa larga e abertura de ventilação coberta com filó de nylon. Triagens foram feitas diariamente para retirada dos insetos adultos que emergiam dos capítulos, sendo suspensas após dois meses de criação, quando havia duas semanas que não emergiam mais insetos. Todos os insetos foram identificados usando literatura especializada, posteriormente, enviados para especialista.

Análise dos dados. análise da relação da riqueza e abundância de espécies com tempo pós- queimada foi feita através do

Tabela 1. Abundância e riqueza de insetos, histórico de fogo (em anos), localização e código das treze áreas amostradas em cerrados na Chapada dos Guimarães - MT no período de abril e maio de 2005.

\begin{tabular}{|c|c|c|c|c|c|c|c|}
\hline \multirow{2}{*}{ Cód. Área } & \multirow{2}{*}{ Área } & \multirow{2}{*}{ Localização } & \multicolumn{2}{|c|}{ Coordenadas } & \multirow{2}{*}{$\begin{array}{l}\text { Tempo pós- } \\
\text { queimada } \\
\text { (anos) }\end{array}$} & \multirow{2}{*}{ Riqueza } & \multirow{2}{*}{ Abundância } \\
\hline & & & Latitude & Longitude & & & \\
\hline $\mathrm{a}$ & Bom Jardim & APA & $15^{\circ}$ o9' $52,1^{\prime \prime} \mathrm{S}$ & $55^{\circ} 51^{\prime} 41,4 ” W$ & 5 & 3 & 18 \\
\hline $\mathrm{b}$ & Colégio Buriti & APA & $15^{\circ} 24^{\prime} 50,8 ” S$ & $55^{\circ} 46^{\prime} 56,4^{\prime \prime} \mathrm{W}$ & 2 & 1 & 3 \\
\hline $\mathrm{c}$ & $\begin{array}{c}\text { Cidade de } \\
\text { Pedra } 1\end{array}$ & PARNA & $15^{\circ} 20^{\prime} 08,6 " \mathrm{~S}$ & $55^{\circ} 47^{\prime} \mathrm{o} 6,7^{\prime \prime} \mathrm{W}$ & 2 & 1 & 1 \\
\hline $\mathrm{d}$ & $\begin{array}{l}\text { Cidade de } \\
\text { Pedra } 2\end{array}$ & PARNA & $15^{\circ} 17^{\prime} 57,8^{\prime \prime S}$ & $55^{\circ} 50^{\prime} 20,8^{\prime \prime} \mathrm{W}$ & 5 & 0 & o \\
\hline e & Fazenda & APA & $15^{\circ} 12^{\prime} 35,1^{\prime \prime} \mathrm{S}$ & $55^{\circ} 41^{\prime} 14,7 ” \mathrm{~W}$ & 15 & 1 & 23 \\
\hline $\mathrm{f}$ & Haras Talismã & PARNA & $15^{\circ} 19^{\prime} 33,2 " \mathrm{~S}$ & $55^{\circ} 52^{\prime} 47,1^{\prime \prime} \mathrm{W}$ & 4 & 1 & 6 \\
\hline $\mathrm{g}$ & Rio Mutuca & PARNA & $15^{\circ} 21^{\prime} 13,6^{\prime \prime} \mathrm{S}$ & $55^{\circ} 58^{\prime}$ o2, 5 ”'W & 3 & 1 & 2 \\
\hline $\mathrm{h}$ & $\begin{array}{c}\text { Chácara Toa } \\
\text { Toa }\end{array}$ & PARNA & $15^{\circ} 20^{\prime} 33,0 " \mathrm{~S}$ & $55^{\circ} 51^{\prime} 21,8^{\prime \prime} \mathrm{W}$ & 5 & 4 & 52 \\
\hline i & Véu de Noiva & PARNA & $15^{\circ} 24^{\prime} 08,9 " S$ & $55^{\circ} 49^{\prime} 40,4^{\prime \prime} \mathrm{W}$ & 1 & 4 & 222 \\
\hline $\mathrm{j}$ & Rio Claro 1 & PARNA & $15^{\circ} 20^{\prime} 14,3 " \mathrm{~S}$ & $55^{\circ} 53^{\prime} 39,3 ” \mathrm{~W}$ & 1 & o & o \\
\hline $\mathrm{k}$ & Rio Claro 2 & PARNA & $15^{\circ} 20^{\prime} 15,1^{\prime \prime} \mathrm{S}$ & $55^{\circ} 53^{\prime} 36,3$ "W & 1 & 2 & 7 \\
\hline 1 & Rio Claro 3 & PARNA & $15^{\circ} 20^{\prime} 55,2 " \mathrm{~S}$ & $55^{\circ} 52^{\prime} 47,2^{\prime \prime} \mathrm{W}$ & 1 & 1 & 7 \\
\hline $\mathrm{m}$ & Morro do Buda & APA & $15^{\circ}$ o6' 03,1”S & $55^{\circ} 56^{\prime}$ o3,6” W & 4 & o & o \\
\hline
\end{tabular}


Coeficiente de correlação de Spearman, após análise de normalidade das variáveis resposta. Uma ordenação direta foi utilizada para análise da distribuição das espécies ao longo do gradiente representado pelo tempo decorrido pós-queimada. A ordem das espécies foi determinada pelo cálculo da média ponderada da abundância de cada espécie em cada área.

\section{RESULTADOS}

Riqueza, Abundância e Ocorrência. Foram coletados 574 indivíduos e 53 morfoespécies de Asteraceae nas 13 áreas amostradas. Emergiram insetos de capítulos coletados em dez das 13 áreas. (Tabela 1). Foram obtidos 341 indivíduos de sete espécies de Tephritidae (Tabela 2). Cecidochares Bezzi e Xanthaciura Hendel foram os gêneros mais bem representados em relação ao número de espécies.

O gênero com maior número de indivíduos foi Xanthaciura. Xanthaciura chrysura (Thomson) foi a única espécie que ocorreu em todas as localidades que emergiram insetos. Esta foi também a espécie mais abundante $(\mathrm{n}=320)$, representando 94\% do total (Tabela 2). A maioria dos insetos (65\%) emergiu de capítulos coletados em apenas uma localidade. Cecidochares connexa (Macquart) foi encontrada em três áreas, Cecidochares spE e Xanthaciura biocellata (Thomson) em duas áreas e Tomoplagia cf. minuta, Trupanea sp1 e Cecidochares fluminensis (Lima) em apenas uma área (Tabela 2).

A maior riqueza de espécies $(S=4)$ foi observada em duas áreas, ambas localizadas dentro do PNCG (áreas h; i). Em 46,0\% das áreas encontramos somente uma espécie $(S=1)$ de Tephritidae (Tabela 2). A riqueza média foi 3,0 $\pm 1,2 \mathrm{SD}$ e a abundância média dos indivíduos emergidos foi 34,1 \pm 64,0 SD.

Riqueza e Abundância em relação ao tempo decorrido pós-queimada. Não houve efeito significativo do tempo decorrido pós-queimada sobre a riqueza de espécies ( $r s=$ -0,0166; P> 0,05) (Tabela 2) e a abundância populacional total $(\mathrm{rs}=0,1292 ; \mathrm{P}>0,05)$ de Tephritidae (Tabela 2).

Os valores de abundância destes insetos apresentaram grandes variações entre áreas com mesmo histórico de fogo. Em áreas que não queimavam há um ano, por exemplo, foram encontrados o menor $(\mathrm{n}=1)$ e o maior $(\mathrm{n}=222)$ número de indivíduos (Tabela 2).

Relação entre o tempo decorrido pós-queimada e a distribuição das espécies. A maioria das espécies ocorreu em áreas que não queimavam havia cinco anos (Figura 1). Apenas uma espécie ocorreu nas áreas que não queimavam havia dois, três, quatro e 15 anos. Portanto, a distribuição de espécies não ocorreu ao longo de um gradiente de tempo pós queimada, indicando que esta perturbação não atuou como agente estruturador dessa comunidade.

\section{DISCUSSÃO}

Riqueza, Abundância e Ocorrência. A riqueza encontrada representa 9\% do total das espécies já descritas no Brasil (PRADO et al. 2002). PRADO et al. (2002) realizaram um trabalho de compilação de Tephritidae no sul esudeste do Brasil e encontraram 80 espécies destes insetos. AlmEida et al. (2006), registraram para o Estado de São Paulo 38 espécies de Tephritidae. Ainda

Tabela 2. Número de indivíduos de cada espécie de Tephritidae emergidos de espécies de Asteraceae coletadas em 13 localidades com históricos de fogo diferentes (em anos) nos cerrados de Chapada dos Guimarães - MT no período de abril e maio de 2005.

\begin{tabular}{|c|c|c|c|c|c|c|c|c|c|c|c|c|c|c|c|}
\hline \multirow[b]{2}{*}{ Espécies de Tephritidae } & \multicolumn{15}{|c|}{ Áreas } \\
\hline & $\mathbf{e}$ & $\mathbf{a}$ & $\mathbf{h}$ & d & $\mathbf{f}$ & $\mathbf{m}$ & $\mathbf{g}$ & $\mathbf{b}$ & $\mathbf{c}$ & $\mathbf{k}$ & 1 & $\mathbf{i}$ & $\mathbf{j}$ & Total & $\begin{array}{c}\text { Freqüência } \\
\text { de ocorrência }\end{array}$ \\
\hline Xanthachiura chrysura & 23 & 14 & 47 & - & 6 & - & 2 & 3 & 1 & 6 & 7 & 211 & - & 320 & 76,92 \\
\hline Cecidochares connexa & - & - & - & - & - & - & - & - & - & 1 & - & 3 & - & 4 & 15,38 \\
\hline Cecidochares sp.E & - & - & 2 & - & - & - & - & - & - & - & - & 7 & - & 9 & 15,38 \\
\hline Xanthachiura biocellata & - & - & 2 & - & - & - & - & - & - & - & - & 1 & - & 3 & 15,38 \\
\hline Cecidochares fluminensis & - & - & 1 & - & - & - & - & - & - & - & - & - & - & 1 & 7,69 \\
\hline Tomoplagia cf. minuta & - & 3 & - & - & - & - & - & - & - & - & - & - & - & 3 & 7,69 \\
\hline Trupanea sp1 & - & 1 & - & - & - & - & - & - & - & - & - & - & - & 1 & 7,69 \\
\hline Abundância & 23 & 18 & 52 & o & 6 & $\mathrm{O}$ & 2 & 3 & 1 & 7 & 7 & 222 & $\mathrm{O}$ & 341 & - \\
\hline Riqueza & 1 & 3 & 4 & $\mathrm{O}$ & 1 & $\mathrm{O}$ & 1 & 1 & 1 & 2 & 1 & 4 & o & - & - \\
\hline
\end{tabular}

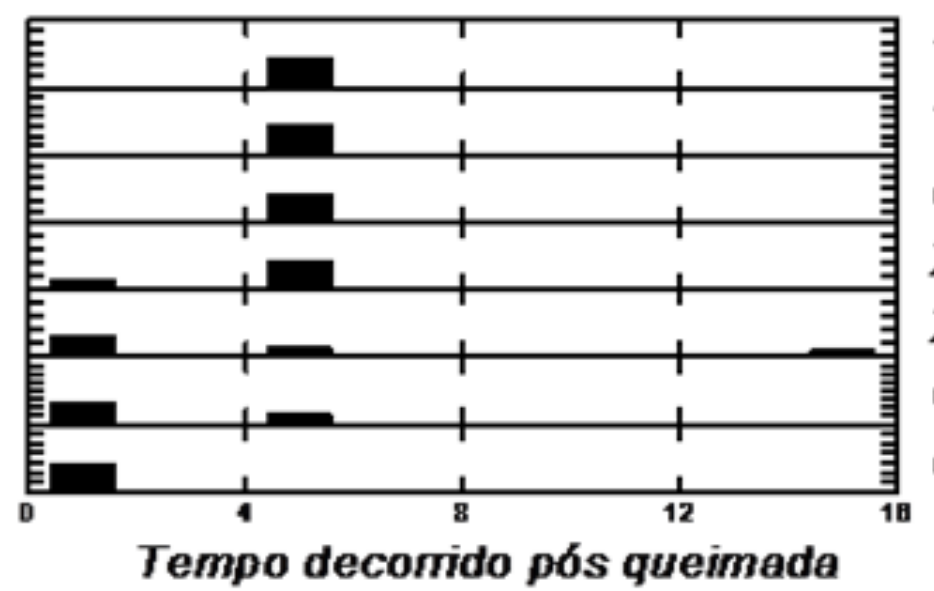

Trupanea sp1

Tomoplagia cf minuta

Cecidochares fluminensis

Xenthachiura biocellate

Xanthachiura chrysura

Cecidochares sp.E

Cecidochares connexa

Tempo decorido pós queimada

Figura 1. Distribuição da abundância relativa de espécies de Tephritidae ao longo do gradiente (tempo decorrido após a última queimada em anos) em cerrados na Chapada dos Guimarães - MT no período de abril e maio de 2005 
em cerrados de São Paulo, KuвотA (2003) encontrou 15 espécies e Diniz (2003) registrou 17 espécies.

A riqueza encontrada foi elevada se compararmos nosso esforço amostral com os demais trabalhos. Prado et al. (2002), amostraram em média quatro áreas de 48 localidades, e a maioria foi visitada mais de uma vez durante os 13 anos de pesquisa. Almeida et al. (2006) visitaram oito áreas uma vez ao ano durante três anos consecutivos. KuвотA (2003) realizou amostragem de capítulos feita por meio de caminhadas ao acaso numa área de 6ha (300 x 200m) e Diniz (2003) coletou os capítulos das plantas hospedeiras com esforço amostral de quatro pessoas-hora em uma área total de 300 x 50m.

Xanthaciura foi o gênero mais representativo, também em estudos nos cerrados do estado de São Paulo (Diniz 2003; DiAs 2003; KuBota 2003). A elevada abundância de X. chrysura foi observada também por Diniz (2003).

Das espécies encontradas somente $X$. chrysura ocorreu em todas as localidades que emergiram insetos, assim como em estudos realizados por Almeida et al. (2006). A alta abundância e ocorrência da espécie em diferentes localidades sugerem que $X$. chrysura possua uma alta tolerância ambiental. Segundo MACEDO (2000), espécies que tenham maiores tolerâncias ambientais apresentariam maiores abundâncias locais e maiores distribuições.

O maior número de espécies de Cecidochares e Xanthaciura tambémfoiobservadopor Diniz(2003)e DiAs(2003). Isto ocorreu devido ao período de amostragem corresponder à época de floração das espécies da tribo Eupatorieae, que são hospedeiras preferenciais destes gêneros (PRADO et al. 2002). No entanto, a fauna de Tephritidae em capítulos de Asteraceae no Brasil é dominada pelo gênero Tomoplagia Coquillett (PRADO et al. 2002; Bonfim et al. 2007), seguida por Xanthaciura e Trupanea Schrank (PRADo et al. 2002).

Riqueza e Abundância em relação ao tempo decorrido pós-queimada. Esperava-se que a riqueza de insetos fosse baixa logo após a passagem do fogo, e que aumentaria nas áreas recentemente queimadas, onde há o favorecimento de gramíneas e herbáceas (KaUfFAman et al. 1994). Conseqüentemente, haveria o aumento das populações de plantas hospedeiras destes insetos. Em áreas onde o fogo não passa por longo período de tempo, haveria o decréscimo das populações de plantas em decorrência do aumento significativo na abundância do componente lenhoso da vegetação (CoutinHo 1990). No entanto, os valores de riqueza destes insetos apresentaram grandes variações entre áreas com mesmo histórico de fogo. Em áreas que não queimavam há um ano, por exemplo, foram encontradas a menor $(S=1)$ e a maior $(S=4)$ riquezas.

Outros fatores podem estar relacionados à riqueza de Tephritidae. A variação da riqueza da comunidade de insetos endófagos de capítulos está relacionada com fatores climáticos, e também com a variação da disponibilidade de capítulos e da riqueza de Asteraceae em flor durante o período de estudo (КивотA 2003). A seqüência de floração das tribos é responsável por sustentar as diferentes comunidades de insetos em seus respectivos períodos de floração. Isto ocorre devido estes endófagos serem monófagos ou oligófagos, alimentando-se preferencialmente dentro de uma tribo ou gênero (PRADO et al. 2002).

Os valores de abundância destes insetos também apresentaram grandes variações entre áreas com mesmo histórico de fogo. Para SwEngel (2001), muitos insetos declinam acentuadamente no período imediato pós-fogo e num período intermediário os efeitos são bastante variáveis. Alguns taxa persistem em número reduzido, outros são controlados pelo efeito e ainda há os insetos que têm sua abundância aumentada após a ocorrência do fogo.

Relação entre o tempo decorrido pós-queimada e a distribuição das espécies. O número de espécies de Tephritidae em áreas não queimadas um ano antes do estudo foi elevado, porém, não mais do que em áreas que estavam sem sofrerem queimadas havia cinco anos. Os resultados sugerem que as espécies levam em torno de cinco anos para conseguirem recolonizar e restabelecer o local afetado pela perturbação.

O número reduzido de espécies encontradas na área que não havia sido queimada há 15 anos poderia ser explicado pela baixa luminosidade em conseqüência da maior presença do componente lenhoso da vegetação devido à ausência do fogo. $\mathrm{O}$ fogo geralmente altera a estrutura da vegetação e composição, bem como a cobertura do terreno (Wilson \& SHAY 1990; НосHкIRCH \& ADORF 2007). Áreas recentemente queimadas são mais abertas e iluminadas (Usher 1992; KIss \& MAGNIN 2005). No entanto, áreas que não queimavam havia dois, três e quatro anos apresentaram mesmo número de espécies. Portanto, a luminosidade por si só também não seria um fator determinante na distribuição das espécies de Tephritidae.

É importante considerar que o número de áreas amostradas para cada tempo decorrido pós-queimada não foi o mesmo. Assim sendo, os resultados obtidos poderiam ser apenas um artefato gerado pela diferença de esforço amostral. Entretanto, áreas com maiores números de amostras (um ano) não foram as mais ricas. Áreas com mesmo número de amostras, como as de dois e as de cinco anos apresentaram diferentes riquezas e composição de insetos. Ou seja, os resultados de abundância e riqueza de espécies não são explicados pela questão amostral.

\section{AGRADECIMENTOS}

À Fundação de Amparo à Pesquisa do Estado de Mato Grosso (FAPEMAT) pelo apoio financeiro à pesquisa. Ao Dr. Mário Almeida Neto pela conferencia e identificação das espécies de Tephritidae e ao Dr. Roberto Lourenço Esteves pela identificação das Asteraceae.

\section{REFERÊNCIAS}

Almeida, A.M., C.R Fonseca, P.I Prado, M. Almeida-Neto, S. Diniz, U. Kubota, M.R. Braun, R.L.G Raimundo, L.A Anjos, T.G. Mendonça, S.M. Futada \& T.M. Lewinsohn, 2005. Diversidade e Ocorrência de Asteraceae em Cerrados de São Paulo. Biota Neotropica, 5: 1-17.

Almeida, A.M., C.R. Fonseca, P.I. Prado, M. Almeida-Neto, S. Diniz, U. Kubota, M.R. Braun, R.L.G.Raimundo, L.A. Anjos, T.G. Mendonça, S.M. Futada \& T.M. Lewinsohn, 2006. Assemblages of Endophagous Insects on Asteraceae in São Paulo Cerrados. Neotropical Entomology, 35: 558-468.

Anderson, R.C., T. Leahy \& S.S. Dhillion, 1989. Numbers and biomass of selected insect groups on burned and unburned sand prairie. American Midland Naturalist, 122: 151-162.

Batalha, M.A. \& W. Mantovani, 2001. Floristic composition of the cerrado in the Pé-de-Gigante reserve (Santa Rita do Passa Quatro, Southeastern Brazil). Acta Botanica Brasil, 15: 289302.

Bock, C.D. \& J.H. Bock, 1991. Response of grasshoppers (Orthoptera: Acrididae) to wildfire in a southeastern Arizona grassland. American Midland Naturalist, 125: 162-167.

Bonfim, D.A., M.A. Uchoa-Fernandes \& M.A.L. Bragança, 2007. Biodiversity of fruit (Diptera, Tephritoidea) in native forests and orchards in two counties of the State of Tocantins, Brazil. Revista Brasileira de Entomologia, 51:2, 217-223.

Bremer, K., 1994. Asteraceae: Cladistics and Classification. Timber Press, Portland, 752 p.

Costa, F.R.C., W.E. Magnusson \& R.C. Luizão, 2005. Mesoscale distribution patterns of Amazonian understorey herbs in relation to topography, soil and watersheds. Journal of Ecology, 93: 863-878.

Coutinho, L.M., 1990. Fire in the ecology of the Brazilian Cerrado. In: Goldammer J.G. (ed.) Fire in the Tropical Biota, SpringerVerlag, Berlin, 82-105 p.

Desouza, L.B.A, V.M Tonello, L. P. Pinto \& R.R. Junior, 2003. Effects of fire on Térmite generic richness in a Savanna-like 
ecosystem ('Cerrado') of Central Brazil. Sociobiology, 42: 639-648.

Dias, A.T.C., 2003. Efeito da disponibilidade de recurso em um sistema com três níveis tróficos. Dissertação (Mestrado em Ecologia) - Instituto de Biologia - Universidade Estadual de Campinas, Campinas- SP.

Didham, R.K., J. Ghazoul, N.E. Stork \& A.J. Davis, 1996. Insect in fragmented forests: a functional approach. Trends in Ecology \& Evolution, 11: 255-260.

Diniz, S., 2003. Diversidade de Asteraceae e de insetos associados e padrões de uso de hospedeiro por Tephritidae (Diptera) em diferentes componentes da paisagem do Estado de São Paulo. Dissertação (Mestrado em Ecologia) - Instituto de Biologia Universidade Estadual de Campinas, Campinas- SP.

Evans, E.W., 1984. Fire as a natural disturbance to grasshopper assemblages of tallgrass prairie. Oikos, 43: 9-16.

Foote, R.H., 1980. Fruit Fly Genera South of the United States. Technical Bulletin 1600. United States Departament of Agriculture, Washington, 79 pp.

Foote, R.H., F.L. Blanc \& A.L. Norrbom, 1993. Handbook of the Fruit Flies (Diptera: Tephritidae) of America North of Mexico. Comstock Publishing Associates, Ithaca, $571 \mathrm{p}$.

Fredericksen, N.J. \& T.S. Fredericksen, 2002. Terrestrial wildlife responses to logging and fire in a Bolivian tropical humid forest. Biodiversity and Conservation, 11: 27-38.

Hochkirch, A. \& F. Adorf, 2007. Effects of prescribed burning and wildfires on Orthoptera in Central European peat bogs. Environmental Conservation, 34: 225-235.

IBAMA, 2007. Ministério do Meio Ambiente - MMA, Instituto do Meio Ambiente e Recursos Naturais Renováveis. Centro Nacional de Prevenção e Combate aos Incêndios Florestais - Prevfogo. Plano operativo de prevenção e combate aos incêndios florestais do Parque Nacional da Chapada dos Guimarães - MT

Kauffman, B.J., D.L. Cummings \& D.E. Ward, 1994. Relationship of fire, biomass and nutrient dynamics along a vegetation gradient in the Brazilian cerrado. Journal of Ecology, 82: 519-531.

Kiss, L. \& F. Magnin, 2005. High resilience of Mediterranean land snail communities to wildfires. Biodiversity and Conservation, 15: 2925-2944.

Klink, C.A., H. S Miranda, M. I. Gonsalez \& K.R.F. Vicentini, 2002. O bioma Cerrado, Site 3. In: Seeliger U.; C. Cardozzo; F. Barbosa (ed.). Os Sites e o Programa Brasileiro de Pesquisas Ecológicas de Longa Duração, 1: 51-68.

Kubota, U., 2003. Fenologia da comunidade de Asteraceae, variação temporal e determinantes locais de riqueza de insetos endófagos de capítulos. Dissertação (Mestrado em Ecologia) - Instituto de Biologia - Universidade Estadual de Campinas, Campinas- SP.

Kubota, U.; R.D. Loyola, A.M. Almeida, D.A. Carvalho \& T.M. Lewinsohn, 2007. Body size and host range co-determine the altitudinal distribution of Neotropical tephritid flies. Global Ecology and Biogeography, 16: 632-639.

Lewinsohn, T.M., 1991. Insects in flower heads of asteraceae in Southeast Brazil. A tropical case study on species richness, In:

\section{Como citar este artigo:}

Fávero, K., L. Bordignon, K. Vecchi Junior \& S. Diniz, 2010. Efeito do Tempo Pós-Queimada Sobre Comunidades de Tephritidae (Diptera) em Áreas de Cerrado na Chapada dos Guimarães - MT. EntomoBrasilis, 3(2): 29-33. www.periodico.ebras.bio.br/ojs
Price P.W.; T.M. Lewinsohn, G.W. Fernandes, \& W.W. Benson (ed.) Plant-Animal Intreractions: Evolutionary Ecology in Tropical and Temperate Regions. Wiley/Interscience, New York, 525-560 p.

Lewinsohn, T.M., P.I.K.L. Prado, V.N Solferini, J. R. Trigo, A.M. Almeida, A.P. Dornelles, M.A.B. Lopes, A.C.C. Macedo \& B.D. Buys, 1997. How feasible is a full-fledged model system for communities? Charting associations of Brazilian Asteraceae and their flowerhead feeders, In: Bentley, B., S.M. Louda \& R. Root (ed.) The Role of Model Systems in Ecological and Evolutionary Research, (10 th Annual Colloquium) Bodega Marine Laboratory, Bodega Bay, California. 31-35 p.

Lewinsohn, T.M. \& T. Roslin, 2008. Four ways towards tropical herbivore megadiversity. Ecology Letters, 11: 398-416.

Macedo, A.C.C., 200o. Variação espacial na densidade, riqueza e composição de espécies de insetos associados a capítulos de Senecio brasiliensis (Asteraceae). Dissertação (Mestrado em Ecologia) - Instituto de Biologia - Universidade Estadual de Campinas, Campinas- SP.

Magnusson, W.E.; A.P. Lim, R. Luizão, F. Luizão, F.R.C Costa, C.V Castilho \& V.F. Kinupp, 2005. Rapeld: Uma modificação do método de Gentry para o levantamento da biodiversidade em áreas. Biota Neotropica, 5: 1-6.

Panzer, R., 2002. Compatibility of prescribed burning with the conservation of insects in small, isolated prairie reserves. Conservation Biology, 16: 1296-1307.

Prado, P.I., A.L. Norrbom \& T.M. Lewinsohn, 2004. New species of Tomoplagia Coquillett (Diptera: Tephritidae) from capitula of Asteraceae in Brazil. Neotropical Entomology, 33: 189211.

Prado, P.I., T. M. Lewinsohn, A.M Almeida, A.L. Norrbom, B.D. Buys, A.C. Macedo \& M.B. Lopes, 2002. The fauna of Tephritidae (Diptera) from capitula of Asteraceae in Brazil. Proceedings of the Entomological Society of Washington, 104: 1007-1028.

Prado, P.I.K.L., T.M. Lewinsohn, 2000. Associações insetoplanta no nível local e regional: Tephritidae e Vernonieae na Serra do Espinhaço, In: Martins R.P., T.M. Lewinsohn \& M.S. Barbeitos. Ecologia e Comportamento de Insetos (Série Oecologia Brasiliensis vol VIII). PPGE-UFRJ, Rio de Janeiro, 405-422 p.

Swengel, A.B., 2001. A literature review of insect responses to fire, compared to other conservation managements of open habitat. Biodiversity and Conservation, 10: 1141-1169.

Usher, M.B., 1992. Management and diversity of arthropods in Calluna heathland. Biodiversity and Conservation, 1: 63-79.

Whelan, R.J., 1995. The Ecology of Fire. Cambridge University Press. 346 pp.

Wilson, S.D. \& J.M. Shay, 1990. Competition, fire, and nutrients in a mixed-grass prairie. Ecology, 71: 1959-1967.

Recebido em: 14/09/2009

Aceito em: 20/02/2010

$* * * * * * * * * * * *$

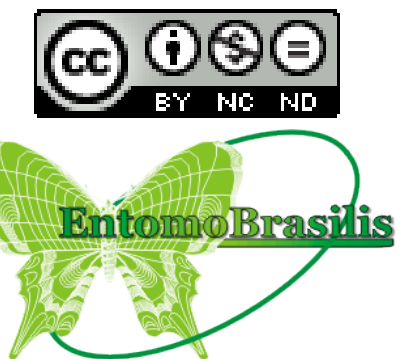

